\title{
Low Temperature Flex-on-Flex Assembly Using Polyvinylidene Fluoride Nanofiber Incorporated Sn58Bi Solder Anisotropic Conductive Films and Vertical Ultrasonic Bonding
}

\author{
Tae-Wan Kim, Kyung-Lim Suk, Sang-Hoon Lee, and Kyung-Wook Paik \\ Department of Materials Science and Engineering, Korea Advanced Institute of Science and Technology (KAIST), \\ 291 Daehak-ro, Yuseong-gu, Daejeon 305-701, Republic of Korea \\ Correspondence should be addressed to Kyung-Lim Suk; stones01@kaist.ac.kr and Sang-Hoon Lee; svl5083@kaist.ac.kr
}

Received 9 August 2013; Accepted 15 November 2013

Academic Editor: Gajanan S. Bhat

Copyright (C) 2013 Tae-Wan Kim et al. This is an open access article distributed under the Creative Commons Attribution License, which permits unrestricted use, distribution, and reproduction in any medium, provided the original work is properly cited.

\begin{abstract}
In this study, solder ball incorporated polyvinylidenefluoride (PVDF) nanofiber was added into the ACF system to overcome short circuit issues of fine pitch flex-on-flex (FOF) assembly. Also, in order to improve the thermal mismatch of the flexible substrate which can lead to electrode misalignment during the bonding process, low melting temperature Sn58Bi solder balls were used with vertical ultrasonic (U/S) bonding method. When performing FOF assembly using PVDF nanofiber/Sn58Bi solder ACF and vertical ultrasonic bonding, PVDF nanofiber/Sn58Bi solder ACFs showed 34\% higher solder capture rate on an electrode compared to conventional Ni ACFs and conventional Sn58Bi solder ACFs. Additionally, PVDF nanofiber/Sn58Bisolder ACFs showed 100\% insulation between neighboring electrodes where conventional Ni ACFs and conventional Sn58Bi solder ACFs showed 75\% and $87.5 \%$ insulation. Other electrical properties such as contact resistance and current handling capability as well as reliability test of PVDF nanofiber/Sn58Bi solder ACFs showed improved results compared to those of conventional Ni ACFs, which proves the formation of stable solder joint of PVDF nanofiber/Sn58Bi solder ACFs.
\end{abstract}

\section{Introduction}

The rapid development of portable electronic products requires miniaturized, multifunctional, and high performance package products. In order to fulfill these requirements, flex-on-flex (FOF) assembly was introduced due to its fine pitch capability and reduced assembly thickness and areas [1].

In various electronic interconnections, anisotropic conductive films (ACFs) are commonly used as an interconnecting material [2-7]. These ACFs are well-known adhesive materials which consist of conductive particles and polymer resin material. However, for conventional ACFs, conductive particle such as gold coated $\mathrm{Ni}$ ball showed insufficient power carrying capability as well as reliability due to the physical contact after bonding. In order to overcome this barrier, metallurgical bonding based solder ACFs with vertical ultrasonic bonding were introduced previously where solder
ACFs showed excellent metallurgical solder joint formation resulting excellent electrical properties and reliability [8-10].

But, when it comes to fine pitch application, solder balls have the tendency to agglomerate between the neighboring electrodes resulting short circuit. Regarding the short circuit issue, nanofiber incorporated ACFs were introduced in the past [11-15] in our group using electrospinning technique [16-19].

In this paper, we will introduce Sn58Bi (melting point: $138^{\circ} \mathrm{C}$ ) incorporated nanofiber/solder ACF. Commonly used solder ball such as SAC305 (96.5\% Sn, 3\% Ag, and 0.5\% $\mathrm{Cu}$ with a melting point of $217^{\circ} \mathrm{C}$ ) requires high bonding temperature $\left(\sim 250^{\circ} \mathrm{C}\right)$ in order to form stable metallurgical joints. This high temperature can cause thermal mismatch of the substrate during bonding process which leads to electrode misalignment. By using Sn58Bi solder ball instead of SAC305 solder ball, it is possible to lower the bonding temperature which will also lower the thermal mismatch between the top 


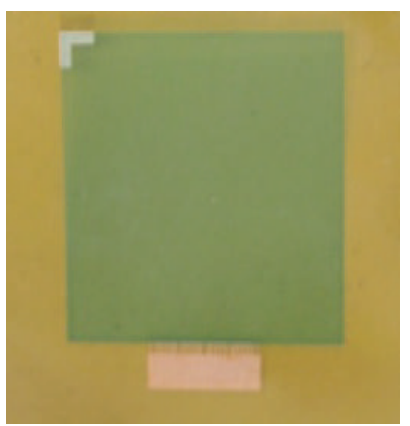

Top substrate

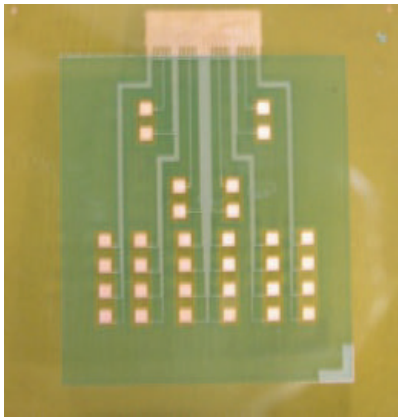

Bottom substrate
FIGURE 1: The images of $100 \mu \mathrm{m}$ pitch top and bottom flexible printed substrates (FPCs).

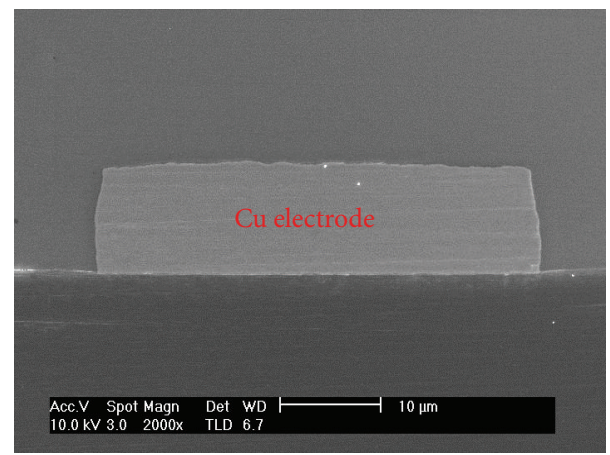

FIGURE 2: The SEM image of the copper electrode of $100 \mu \mathrm{m}$ pitch FPC.

and bottom substrate as well as forming stable solder joint in fine pitch FOF assembly.

\section{Experimental}

\subsection{Material Preparation and Equipment}

2.1.1. Test Vehicle Preparation. Polyimide base $100 \mu \mathrm{m}$ pitch flexible printed circuits (FPCs) were designed as shown in Figure 1. Both top and bottom flexible substrates contain 96 electrodes and the height of each electrode was around $12 \mu \mathrm{m}$ shown in Figure 2. The main bonding area of the FPC was $2 \mathrm{~mm} \times 10 \mathrm{~mm}$. The electrodes of the FPCs were copper electrodes. The copper electrodes were then treated with $100 \mathrm{~nm}$ thick organic solderability preservative (OSP) material in order to prevent any additional oxidation. The bottom FPC contains six contact resistance measurement points and four insulation resistance measurement points.

\subsubsection{PVDF Nanofiber/Sn58Bi Solder ACF Formation.} Nanofibers containing Sn58Bi solder balls were electrospun using the electrospinning equipment shown in Figure 3. Electrospinning is a common method used to produce nanofibers, where voltage is applied to the syringe needle generating a charge at the tip of the needle. As the polymer solution is charged, this will act as a driving force for the polymer solution to migrate from the tip of the needle to

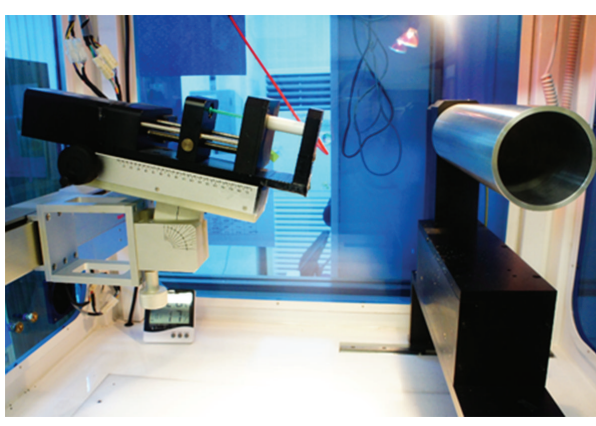

FIGURE 3: The Image of the electrospinning apparatus.

the ground collector producing nanofibers. The nanofiber diameter can be controlled by changing the polymer concentration, applied voltage, working distance, pump rate, and so forth. The compositions of the ACF materials are shown in Table 1. For the optimized PVDF nanofiber/solder ACF, the polymer solution consisted of 15 wt. $\%$ of PVDF, $42 \mathrm{wt} . \%$ dimethylacetamide (DMAC), and $28 \mathrm{wt} . \%$ acetone along with 15 wt.\% Sn58Bi solder balls. After the polymer solution was well mixed with solder balls, the mixed polymer solution was then transported to the syringe followed by the electrospinning process. The PVDF nanofiber was electrospun at $10 \mathrm{KV}$ while working distance, pumping rate, and needle diameter were fixed as $10 \mathrm{~cm}, 20 \mu \mathrm{L} / \mathrm{min}$, and $250 \mu \mathrm{m}$. Then the Sn58Bi solder ball incorporated PVDF nanofiber layer was fabricated and the fiber layer was laminated between two nonconductive films (NCFs) with a thickness of $10 \mu \mathrm{m}$ using the roll and vacuum laminator resulting in final PVDF nanofiber/Sn58Bi solder ACFs with a thickness of $25 \mu \mathrm{m}$.

2.1.3. Vertical Ultrasonic Bonding Apparatus. Solder ball normally contains native oxide, which prevents solder joint to form well. By applying vertical ultrasonic bonding, this native oxide layer can be broken during the bonding process. The vertical ultrasonic (U/S) bonding apparatus was developed previously, and Figure 4(a) shows the one used in this experiment. The longitudinal vibration frequency of the bonder was $40 \mathrm{KHz}$ and the output power was $400 \mathrm{~W}$. Also, the $\mathrm{U} / \mathrm{S}$ vibration amplitude ranged from 4 to $13 \mu \mathrm{m}$. The U/S bonding apparatus have 3 important regions: bonding region, preparation region, and aligning region. The U/S vibration is applied at room temperature and the vibration is mainly applied on the bonding horn located in the bonding region shown in Figure 4(b). The size of the U/S horn was $22 \mathrm{~mm} \times$ $3 \mathrm{~mm}$ which covers the ACF bonding area of FOF assembly. And $200 \mu \mathrm{m}$ thick silicone interposer was placed between the horn and the FPC. The schematic of the ultrasonic bonding process is shown in Figure 5.

2.2. Optimization of Vertical Ultrasonic Bonding Condition. In this experiment, the bonding condition is divided into prebonding condition and main bonding condition. The bonding conditions were decided based on the resin curing temperature, Sn58Bi solder ball melting temperature, and 
TABLE 1: The material composition of conventional Ni ACFs, conventional Sn58Bi solder ACFs, and PVDF nanofiber/Sn58Bi solder ACFs.

\begin{tabular}{lccc}
\hline ACF type & Conventional ACF & Solder ACF & Nanofiber solder ACF \\
Conductive particle content $($ wt.\%) & $30 \mathrm{wt} \% \mathrm{Ni}$ ball & $20 \mathrm{wt} . \%$ Sn58Bi solder ball & 15 wt.\% Sn58Bi solder ball \\
Conductive particle size $(\mu \mathrm{m})$ & $8 \mu \mathrm{m}$ & $5 \sim 15 \mu \mathrm{m}$ & $5 \sim 15 \mu \mathrm{m}$ \\
Nanofiber material & - & - & PVDF \\
Resin type & Epoxy & Epoxy & Epoxy \\
\hline
\end{tabular}

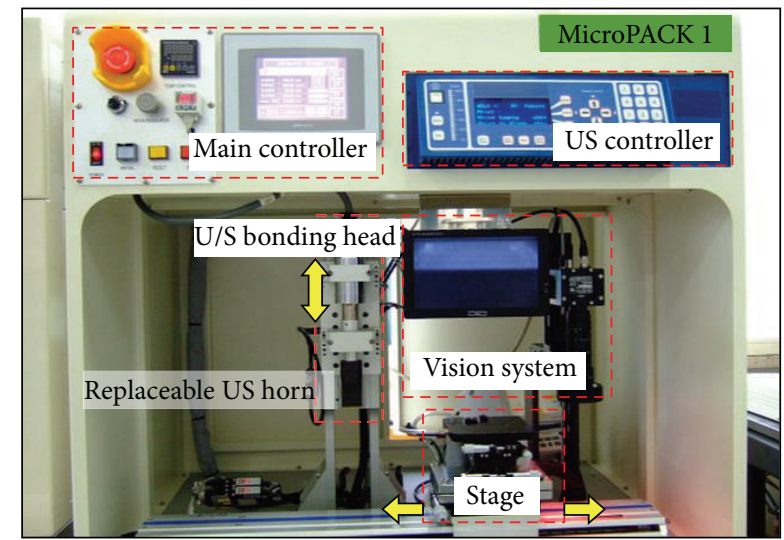

(a)

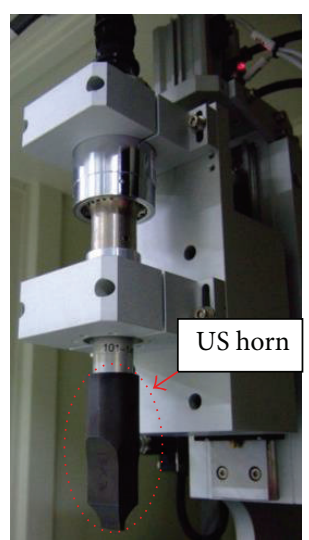

(b)

FIGURE 4: The image of (a) U/S bonding apparatus and (b) U/S horn for FOF bonding.

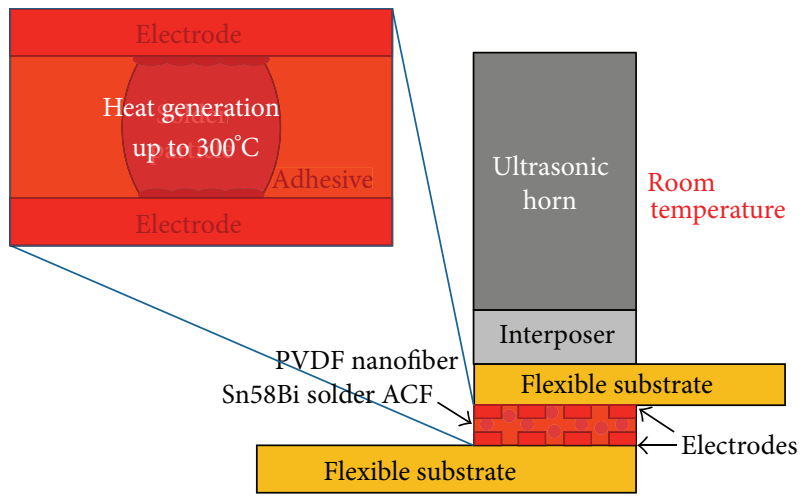

Figure 5: The schematic image of the ultrasonic FOF bonding process.

PVDF melting temperature which was determined by the DSC curve. The prebonding condition was selected so that the conductive particles were captured between the top and bottom electrodes before the main bonding preventing any resin capture during the main bonding process. The main bonding condition was selected so that the solder ball melting occurs while fully curing the resin. Lee et al. performed FOF assembly using $250^{\circ} \mathrm{C}$ as a bonding temperature due to the use of SAC305 solder ball which has a melting point of $217^{\circ} \mathrm{C}$. However, high bonding temperature can result high thermal mismatch resulting electrode misalignment. Thus, in this experiment, both $200^{\circ} \mathrm{C}$ and $250^{\circ} \mathrm{C}$ were considered as bonding temperature candidates for comparison. For comparison, the main bonding pressure and time was fixed to
$3 \mathrm{MPa} 5 \mathrm{sec}$ bonding. Thermal mismatch between the top and bottom FPCs was observed through the optical microscope. In order to measure the total misalignment length one side of the electrode was aligned perfectly while the other side is misaligned. The misalignment value was then compared with the dimensional change of the FPC using the thermal mechanical analysis (TMA) in order to confirm the bonding temperature effect on the thermal mismatch. In addition, the degree of curing was measured through the Fourier transform infrared spectroscopy (FT-IR).

\subsection{Characterization of Nanofiber Effects on ACF Joint Properties}

2.3.1. Conductive Particle Movement Analysis. During the prebonding process, conductive particles tend to move due to polymer resin flow. The conductive particle movements were observed before and after prebonding through the microscopic image. Since it is difficult to observe the conductive particles directly through the metal electrodes using the optical microscope, we use a $26 \mu \mathrm{m}$ thick transparent PI film on one side and $100 \mu \mathrm{m}$ pitch FPC on the other side in order to observe the particle movement through the microscope. The number of conductive particles for conventional Ni ACFs, conventional Sn58Bi solder ACFs, and PVDF nanofiber/Sn58Bi solder ACFs was counted and recorded in order to confirm the nanofiber effect on the solder movement. The number of conductive particles captured was then converted to the particle capture rate for comparison. 


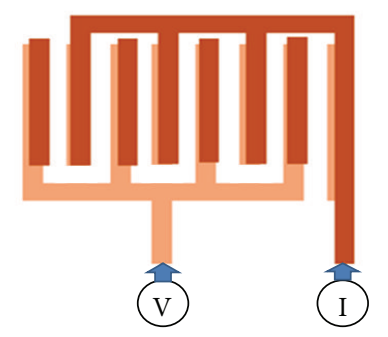

Circuit structure for insulation resistance

(a)

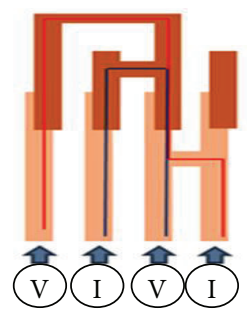

4-point structure for bump contact resistance measurement

(b)

Figure 6: Test fixtures of measuring (a) insulation resistance and (b) contact resistance.

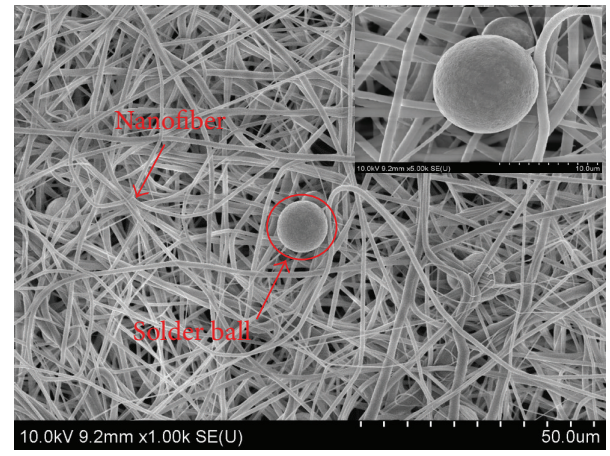

FIGURE 7: The SEM image of Sn58Bi incorporated PVDF nanofiber layer.

2.3.2. Electrical Property Analysis. As mentioned in the conductive movement analysis, conductive particles migrate due to the polymer resin flow during the bonding process causing conductive particles to agglomerate between the neighboring electrodes. For finer pitch applications, this bridging of agglomerated conductive particles may result in electrical short circuit between the electrodes. The insulation rate is the exact opposite of short circuit. When the insulation rate is $100 \%$, this means that there are no short circuits. The insulation property and contact resistances of conventional $\mathrm{Ni}$ ACFs, conventional Sn58Bi solder ACFs, and PVDF nanofiber Sn58Bi solder ACFs were measured by the test fixture shown in Figure 6.

2.3.3. ACF Joint Analysis. After the FPCs were bonded by vertical ultrasonic bonding method, the ACF joints were observed through the scanning electron microscope (SEM) in order to confirm whether stable metallurgical joint forms between the conductive particle and the electrodes of FPCs.

\subsection{Reliability Evaluations on Vertical Ultrasonic Bonded ACF Joints}

2.4.1. Current Carrying Capability. The current carrying capability was determined by measuring the current while applying voltage to the FOF assembly and increasing it gradually using the DC power supply. The voltage was increased by the increment of $0.1 \mathrm{~V}$ after each measurement. The measurement was continued until the current dropped to zero. This allows us to determine the maximum amount of electrical current of the bonded FOF assembly.

2.4.2. Pressure Cooker Test. Lastly, the pressure cooker test (PCT) was performed in order to evaluate the reliability of the FOF assembly using the nanofiber Sn58Bi solder ACFs and U/S bonding and compare this with that of conventional $\mathrm{Ni}$ ACFs and conventional Sn58Bi solder ACFs. The test was performed at $2 \mathrm{~atm}, 121^{\circ} \mathrm{C}, 100 \% \mathrm{RH}$ for 48 hours. The contact resistances were measured for every 16 hours to confirm any electrical open circuit.

\section{Results and Discussion}

3.1. PVDF Nanofiber/Sn58Bi Solder ACF Fabrication. The PVDF nanofiber/Sn58Bi solder ACF was produced first by obtaining PVDF nanofibers through electrospinning technique. As mentioned in the experimental section, the concentration of the polymer solution was optimized and then electrospun at an optimized spinning condition to produce solder ball incorporated PVDF nanofiber. The PVDF nanofiber containing Sn58Bi solder ball is shown in Figure 7. It was observed that PVDF nanofiber completely covers the Sn58Bi solder ball. After producing the PVDF nanofiber, the nanofiber layer was laminated between two $10 \mu \mathrm{m}$ thick NCF films using a roll and vacuum laminator as mentioned before.

\subsection{Optimization of Vertical Ultrasonic Bonding Condition.} The DSC curves showing the resin curing temperature, Sn58Bi solder ball melting temperature, and PVDF melting temperature are shown in Figure 8. Resin curing shows exothermic reaction where solder melting and polymer melting show endothermic reaction. The prebonding was performed below the resin curing temperature $\left(\leq 80^{\circ} \mathrm{C}\right)$ allowing the resin to flow thoroughly before the main bonding process where the solder wetting occurs. The prebonding condition was optimized as $6 \mathrm{MPa}$ pressure at $78^{\circ} \mathrm{C}$ for 10 seconds where it showed sufficient resin flow. The crosssection image after the prebonding condition is shown in 


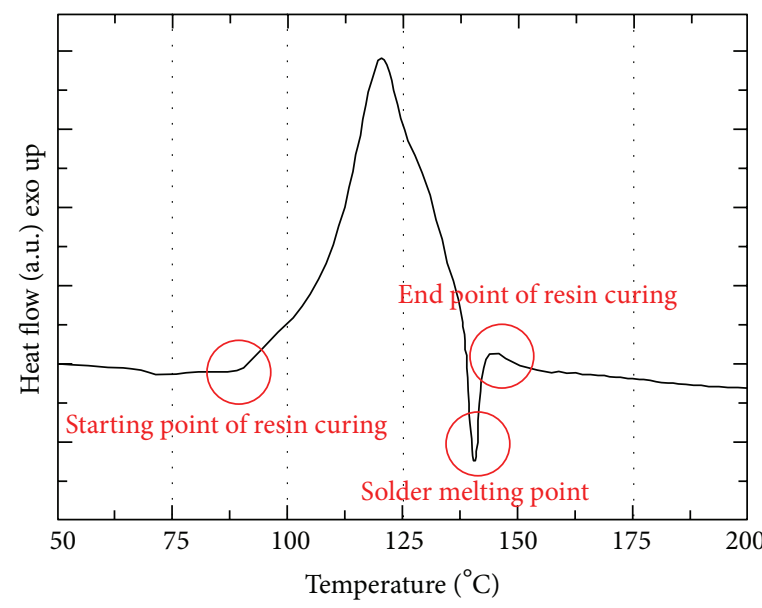

(a)

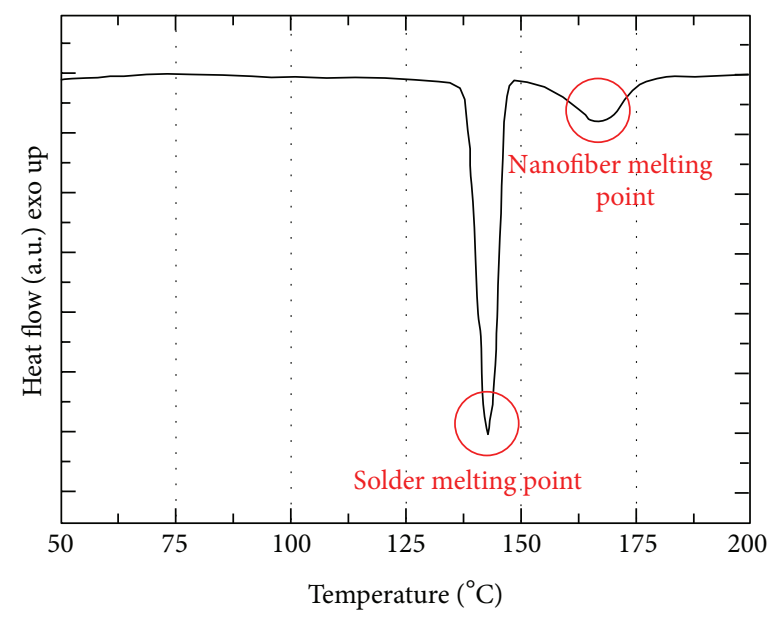

(b)

FIGURE 8: The DSC curve of (a) epoxy base resin containing Sn58Bi solder balls and (b) PVDF nanofiber containing Sn58Bi solder balls.

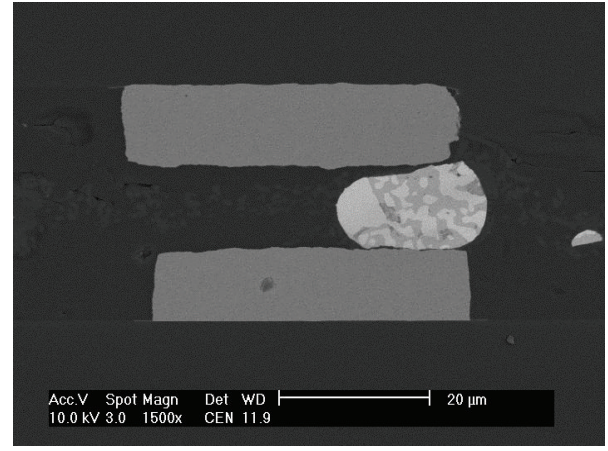

FIGURE 9: The SEM image of joint formation area after the prebonding condition.

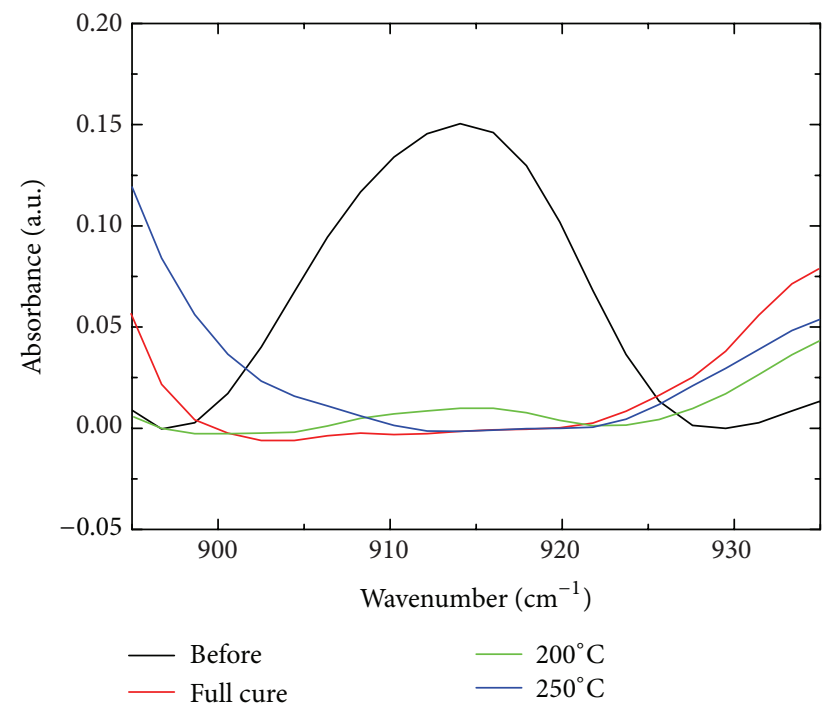

FIGURE 10: The FT-IR results of $200^{\circ} \mathrm{C}$ and $250^{\circ} \mathrm{C}$ bonding temperatures.

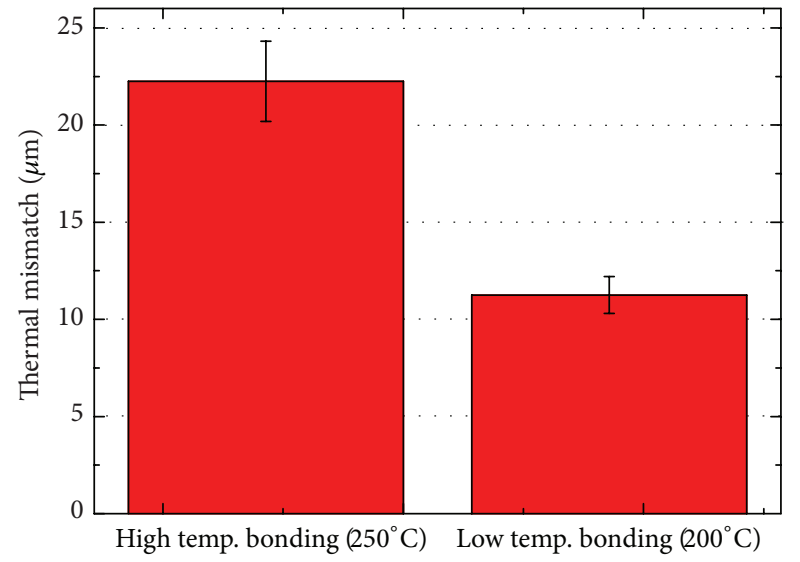

FIgURE 11: The thermal mismatch results of $200^{\circ} \mathrm{C}$ and $250^{\circ} \mathrm{C}$ bonding temperature.

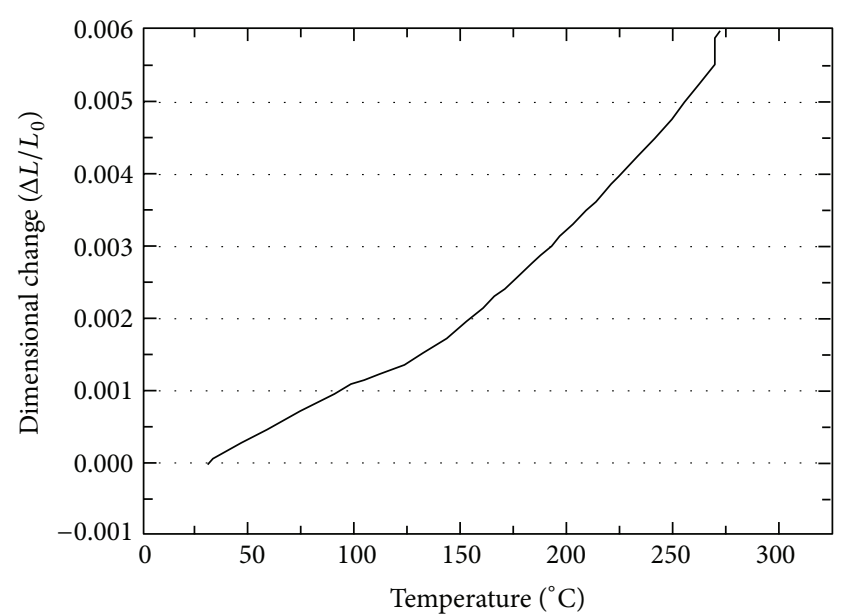

FIGURE 12: The dimensional change curve of FPC substrate using TMA. 


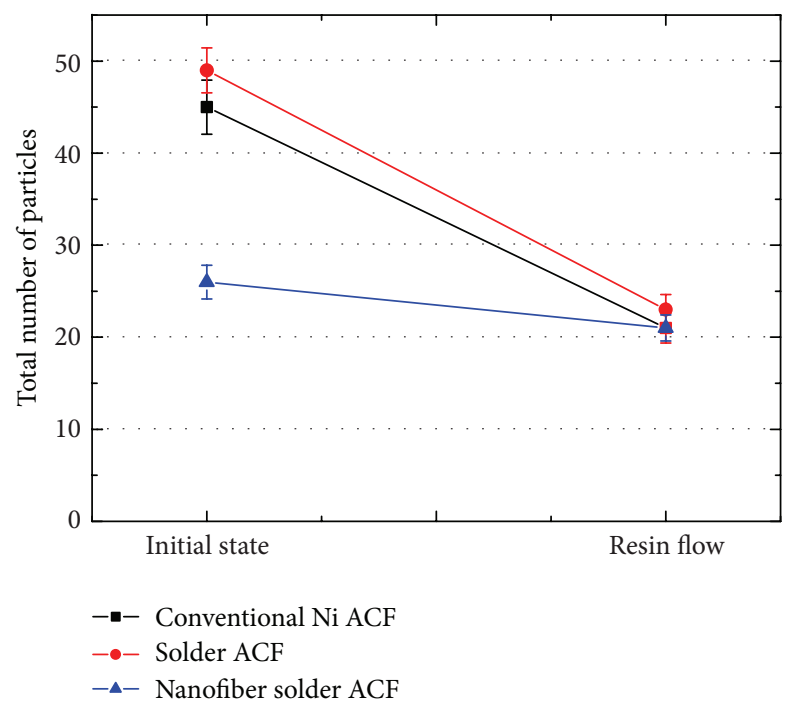

(a)

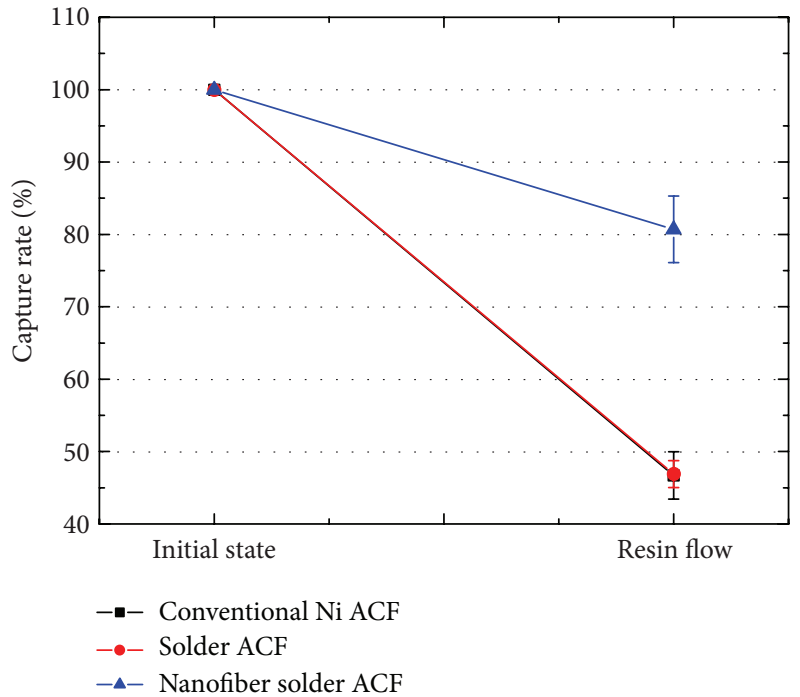

(b)

FIGURE 13: The conductive particle movement analysis results of conventional Ni ACFs, conventional Sn58Bi solder ACFs, and PVDF nanofiber/Sn58Bi solder ACFs. (a) Number of conductive particles on the electrode and (b) conductive particle capture rates before and after bonding.
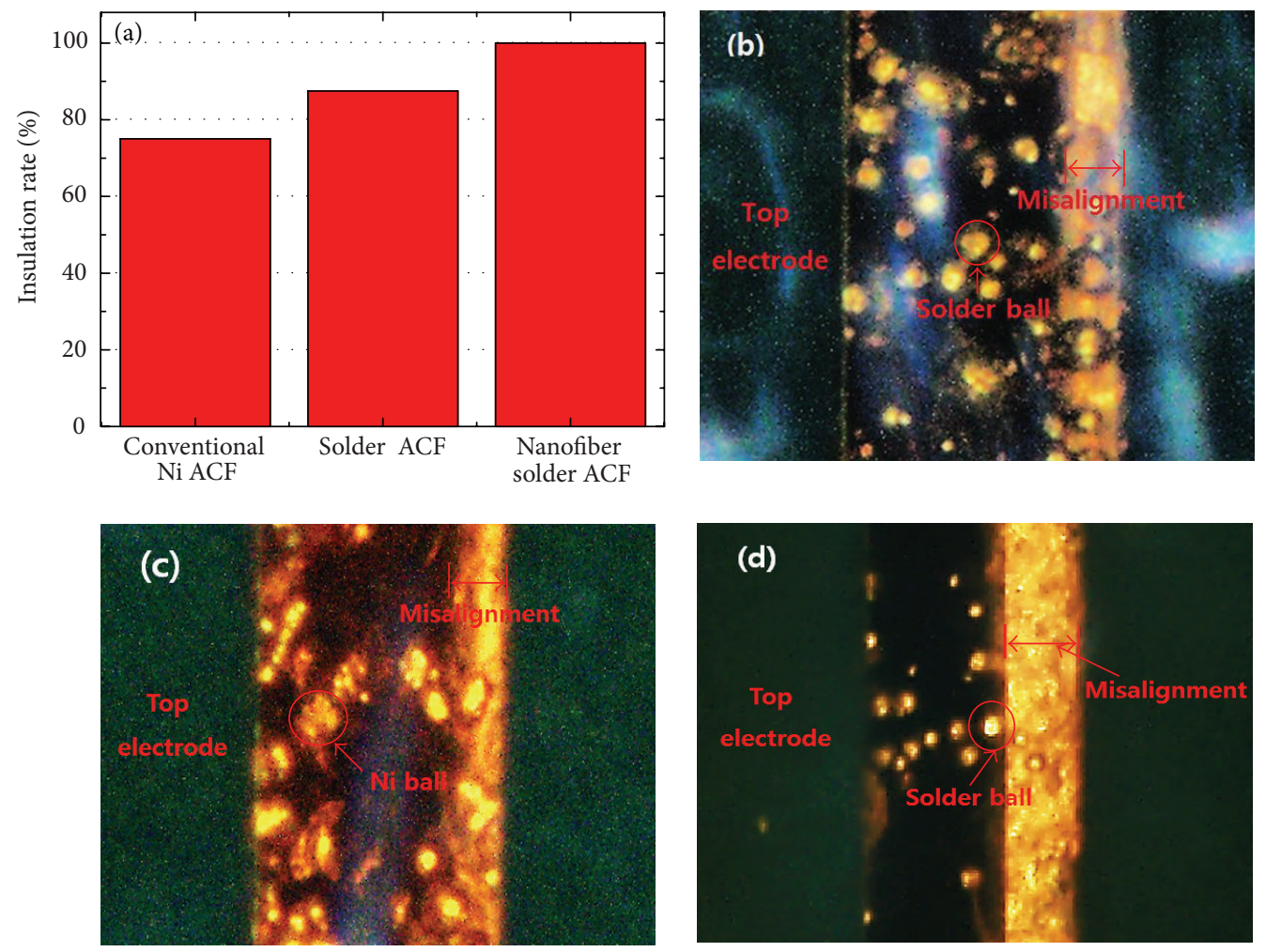

FIGURE 14: (a) The insulation resistance results of conventional Ni ACFs, conventional Sn58Bi solder ACFs, and PVDF nanofiber/Sn58Bi solder ACFs. (b) The optical image of the short circuit region between the neighboring electrodes of the conventional Sn58Bi solder ACFs. (c) The optical image of the short circuit region between the neighboring electrodes of the conventional Ni ACFs. (d) The optical image of the neighboring electrodes of PVDF nanofiber/Sn58Bi solder ACFs. 


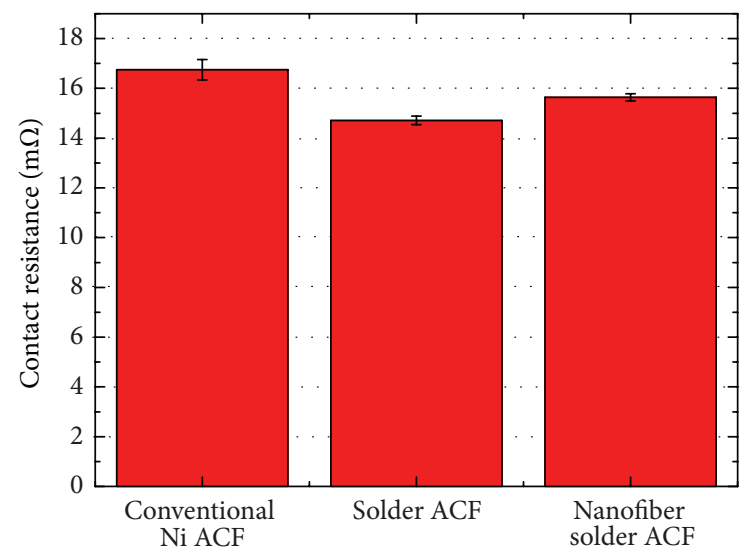

FIgURE 15: The contact resistance of conventional Ni ACFs, conventional Sn58Bi solder ACFs, and PVDF nanofiber/Sn58Bi solder ACFs.

Figure 9. It is shown that the film thickness was reduced down to $10 \mu \mathrm{m}$, where solder ball is closely in contact with the top and bottom electrodes. And then the main bonding was performed to melt the Sn58Bi solder ball and PVDF nanofiber and cure ACF resin finally. As mentioned before for comparison the main bonding pressure and time were set to $3 \mathrm{MPa} 5 \mathrm{sec}$ bonding for comparison. In order to select the main bonding temperature, degree of curing and thermal mismatch were measured. The FT-IR results for $200^{\circ} \mathrm{C}$ and $250^{\circ} \mathrm{C}$ bonded samples are shown in Figure 10. As the resin is cured, the integrated area near $915 \mathrm{~cm}^{-1}$ tends to decrease. For both $200^{\circ} \mathrm{C}$ and $250^{\circ} \mathrm{C}$ bonded samples, more than $90 \%$ resin curing occurred. However when observing the PI film thermal mismatch result after the main bonding process shown in Figure 11, $250^{\circ} \mathrm{C}$ bonded sample showed an average thermal mismatch value of $22 \mu \mathrm{m}$ where $200^{\circ} \mathrm{C}$ bonded sample showed an average thermal mismatch value of $11 \mu \mathrm{m}$. Since the top and bottom FPCs are of the same material, it is expected that the misalignment is due to the thermal gradient. When observing through the thermodetector, the temperature difference between the top flex and the bottom flex was around $150^{\circ} \mathrm{C}$. The misalignment values were compared with the dimensional change results of FPCs using the thermal mechanical analysis (TMA) shown in Figure 12. By lowering the bonding temperature to $200^{\circ} \mathrm{C}$, the thermal mismatch between the top and bottom FPC was lowered which eventually improved the electrode misalignment.

\subsection{Characterization of Nanofiber Effects on ACF Joint Properties}

3.3.1. Conductive Particle Movement Analysis. The results of conductive particle movement analysis are shown in Figure 13. For conventional $\mathrm{Ni} \mathrm{ACF}$ and conventional Sn58Bi solder ACFs, the number of conductive particles located on the electrode area before prebonding was 45 and 49 for $10000 \mu \mathrm{m}^{2}$ area. However, after the prebonding process, the number of conductive particles reduced down to 21 and 23 resulting $47 \%$ capture rate for both ACFs. In contrast, for the PVDF nanofiber/Sn58Bi solder ACFs, 26 solder balls were counted before the prebonding process, and 21 solder balls were captured after the prebonding process, resulting in $81 \%$ capture rate. By using PVDF nanofiber, the conductive particle capture rate was increased by $33 \%$ compared with the conventional Ni ACFs and conventional Sn58Bi solder ACFs because of the suppression of solder ball movement during the prebonding process.

\subsubsection{Electrical Property Analysis. PVDF nanofiber forms} an insulation coating around the solder ball acting as an insulation layer. The insulation property results for conventional Ni ACFs, conventional Sn58Bi solder ACFs, and PVDF nanofiber/Sn58Bi solder ACFs are shown in Figure 14(a). For conventional Ni ACFs and conventional Sn58Bi solder ACFs, conductive particles tend to agglomerate between the electrodes resulting in short circuit after the bonding process. Conventional Ni ACFs and conventional Sn58Bi solder ACFs showed $75 \%$ and $87.5 \%$ insulation rate, while no short circuit was observed for the PVDF nanofiber/Sn58Bi solder ACFs, showing $100 \%$ insulation property which in other words is $0 \%$ short circuit. The optical images of the short circuit region are shown in Figures 14(b) and 14(c). Also, the optical image for the PVDF nanofiber/Sn58Bi solder ACF is shown in Figure 14(d). It was shown that, even though the solder balls tend to agglomerate, the solder ball showed no direct contact due to the nanofiber. This result clearly proves the excellent insulation property of PVDF nanofiber/Sn58Bi solder ACFs compared with conventional $\mathrm{Ni}$ ACFs and conventional Sn58Bi solder ACFs. In addition, the average contact resistances of conventional Ni ACFs, conventional Sn58Bi solder ACFs, and PVDF nanofiber Sn58Bi solder ACFs were $16.7 \mathrm{~m} \Omega, 14.7 \mathrm{~m} \Omega$, and $15.6 \mathrm{~m} \Omega$, respectively, as shown in Figure 15. For conventional Ni ACFs, the Ni ball forms a physical contact with the electrodes preserving its shape, while, for conventional Sn58Bi solder ACFs and PVDF nanofiber/Sn58Bi solder ACFs, the solder ball spreads out forming a metallurgical joint with the electrodes allowing larger contact area. This is why conventional Ni ACF showed the highest contact resistance.

3.3.3. ACF Joint Analysis. The conductive particles form contact joints which act as an electrical pathway. The SEM images for conventional Ni ACFs, Sn58Bi conventional solder ACFs, and PVDF nanofiber/Sn58Bi solder ACFs joints are shown in Figure 16. It is shown that both conventional Sn58Bi solder ACFs and PVDF nanofiber/Sn58Bi solder ACFs form excellent solder metallurgical joint which forms $\mathrm{Cu}-\mathrm{Sn}$ intermetallic compounds (IMC) layer where conventional $\mathrm{Ni}$ ACFs only form physical contact resulting less stable joints. Also, the contact areas for conventional Sn58Bi solder ACF and PVDF nanofiber/Sn58Bi solder ACF joints were larger than that for the conventional $\mathrm{Ni}$ ACFs resulting stable contact resistances as well as good reliability explained later. 


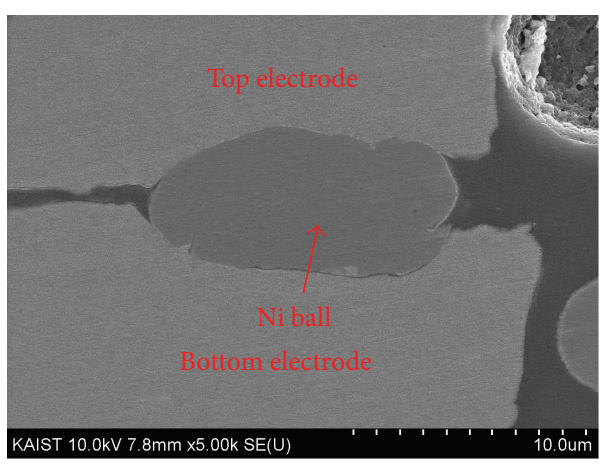

(a)

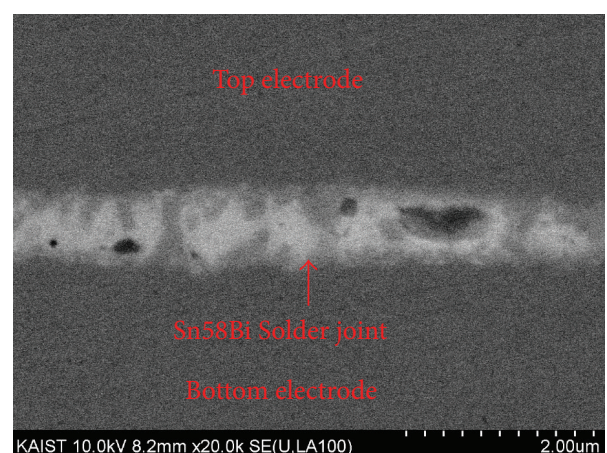

(b)

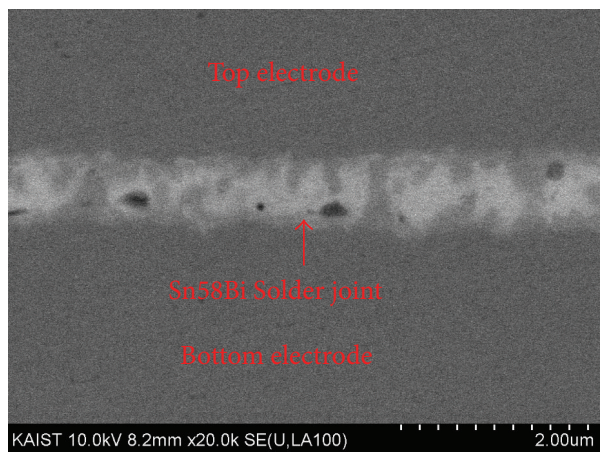

(c)

FIGURE 16: The SEM images of solder ball contact joints of (a) conventional Ni ACF joint, (b) conventional Sn58Bi solder ACF joint, and (c) PVDF nanofiber/Sn58Bi solder ACF joint.

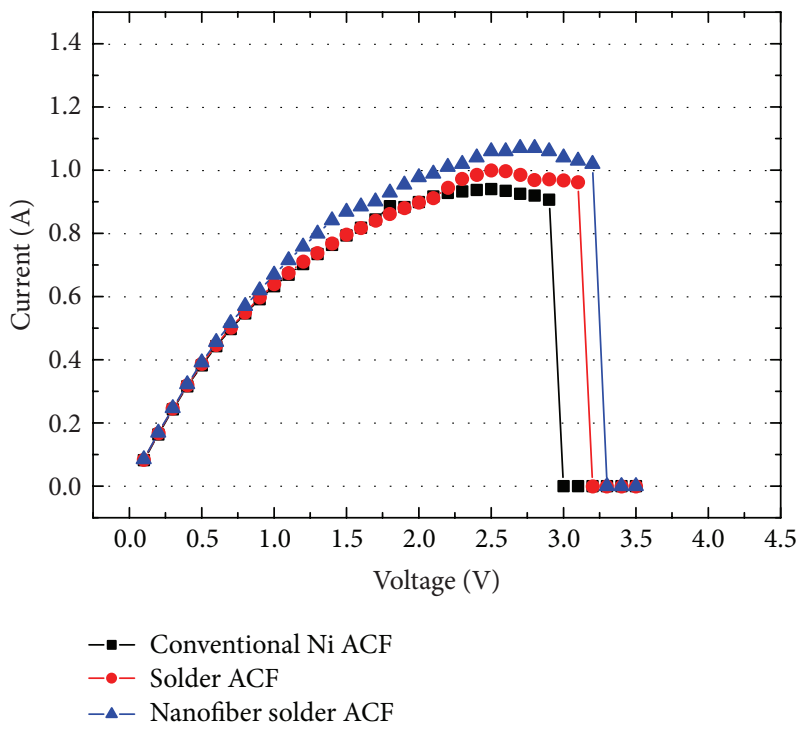

FIGURE 17: The power carrying capability of conventional Ni ACFs, conventional Sn58Bi solder ACFs, and PVDF nanofiber Sn58Bi solder ACFs.

\subsection{Reliability Evaluations on Vertical Ultrasonic Bonded ACF Joints}

3.4.1. Current Carrying Capability. The current carrying capability results of conventional Ni ACFs, conventional
Sn58Bi solder ACFs, and PVDF nanofiber/Sn58Bi solder ACFs are shown in Figure 17. Conventional Ni ACFs were able to carry $0.94 \mathrm{~A}$ at 2.9 volts bias where conventional Sn58Bi solder ACFs and PVDF nanofiber/Sn58Bi solder ACFs were able to carry near $0.99 \mathrm{~A}$ and $1.07 \mathrm{~A}$ at 3.1 and 3.2 volts bias. For conventional Sn58Bi solder ACFs and PVDF nanofiber/Sn58Bi solder ACFs, higher solder joint contact area was observed resulting higher current carrying capability compared with the conventional Ni ACF. However, the main failure occurred at the FPC line damage rather than the solder joint failure. Still it was shown that PVDF nanofiber Sn58Bi solder ACFs have excellent current carrying capability compared to that of the conventional Ni ACF.

3.4.2. Pressure Cooker Test. Joint stability of the FOF assembly ACF joints was tested by a pressure cooker test (PCT). The pressure cooker test (PCT) results of conventional Ni ACFs, conventional Sn58Bi solder ACFs, and PVDF nanofiber/Sn58Bi solder ACFs are shown in Figure 18. For both Sn58Bi solder ACFs and PVDF nanofiber/Sn58Bi solder ACFs, no open circuits were observed even after 48 hours showing stable joint formation where conventional Ni ACFs showed open circuit even after 16 hours of PCT. This is due to the unstable joint stability of the conventional Ni ACF which forms a physical contact between the Ni ball and the electrodes. 


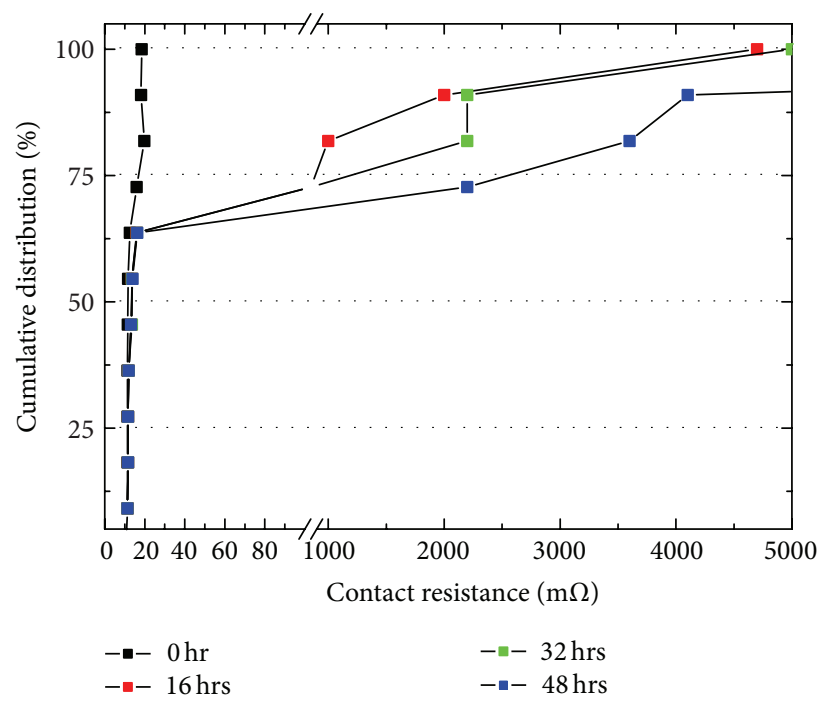

(a)

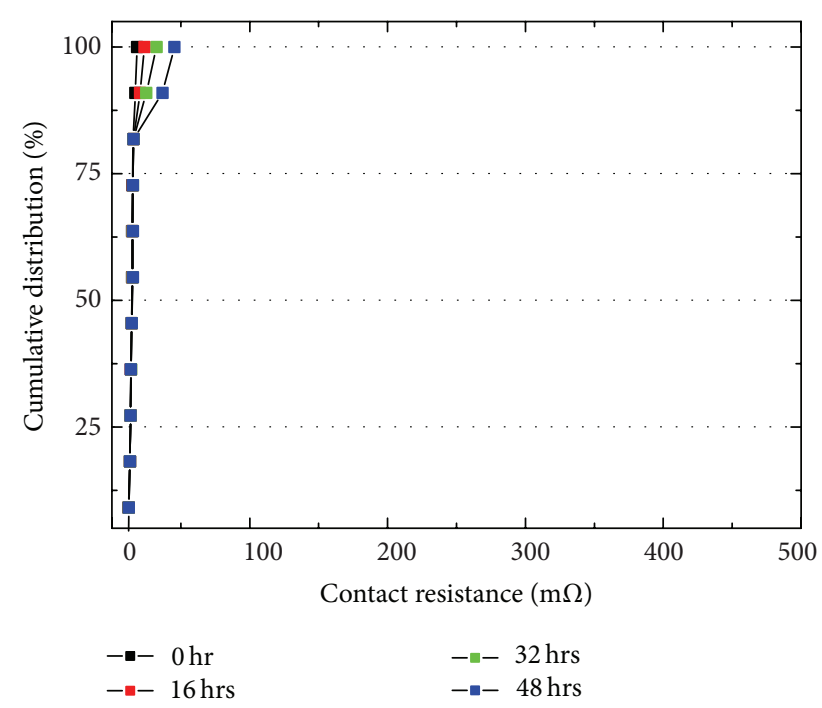

(b)

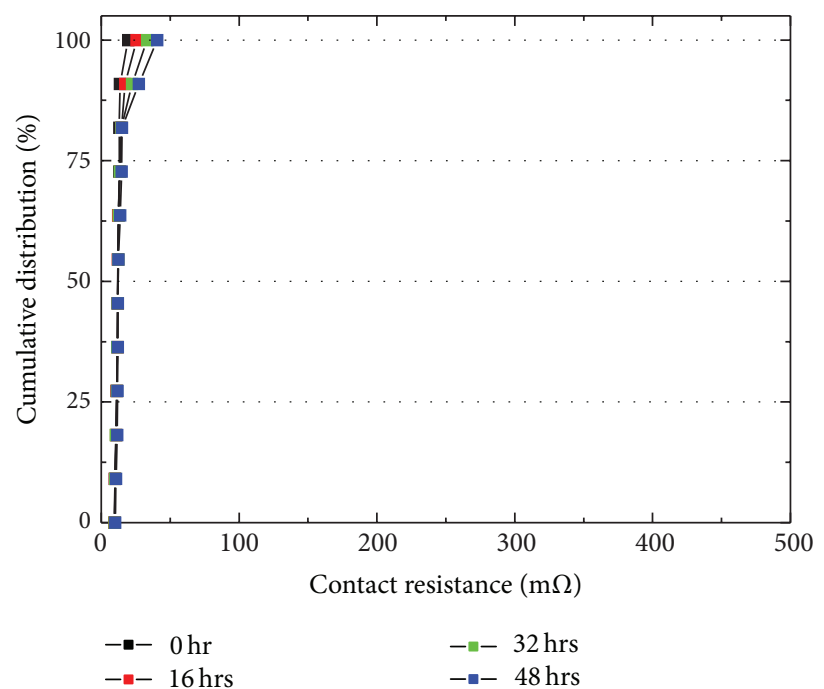

(c)

FiguRE 18: Pressure cooker test results of (a) conventional Ni ACFs, (b) conventional Sn58Bi solder ACFs, and (c) PVDF nanofiber/Sn58Bi solder ACFs.

\section{Conclusion}

Fine pitch flex-on-flex (FOF) assembly was successfully demonstrated using PVDF nanofiber/Sn58Bi solder ACFs and vertical ultrasonic bonding method. By using Sn58Bi solder ball instead of SAC305 solder ball, the bonding temperature was reduced from $250^{\circ} \mathrm{C}$ to $200^{\circ} \mathrm{C}$ resulting improved thermal mismatch. When looking at the conductive particle movement analysis after the bonding process, PVDF nanofiber/Sn58Bi solder ACFs showed 33\% capture rate improvement compared with the conventional $\mathrm{Ni}$ ACFs and conventional Sn58Bi solder ACFs. Also, PVDF nanofiber/Sn58Bi solder ACFs showed 100\% insulation property. It is clearly shown that PVDF nanofiber successfully suppresses the conductive solder ball movement and acts as an insulating layer around the solder ball. When observing the reliability results, conventional Sn58Bi solder ACFs and PVDF nanofiber/Sn58Bi solder ACFs showed preferable reliability results compared to those of conventional Ni ACFs. This is because conventional Sn58Bi solder ACFs and PVDF nanofiber/Sn58Bi solder ACFs contain stable metallurgical solder joints along with the high contact area.

Overall, PVDF nanofiber/Sn58Bi solder ACFs showed excellent solder movement suppressing capability as well as insulation property while lowering the bonding temperature improving the thermal mismatch which eventually improves the electrode misalignment for fine pitch FOF assembly. Currently, the bonding temperature was set to $200^{\circ} \mathrm{C}$ due to the melting point of the nanofiber polymer material and resin curing. If we select a nanofiber polymer material with a low 
melting point (below $150^{\circ} \mathrm{C}$ ) and also use a fast curing resin, it is possible to lower the bonding temperature even down to $150^{\circ} \mathrm{C}$. However, more research needs to done to meet the right mechanical properties and melting temperature of the nanofiber polymer material in order to lower the bonding temperature. This nanofiber solder ACF technology will provide great potential use for future advanced fine pitch electronic assemblies.

\section{References}

[1] C. Beelen-Hendrikx and M. Verguld, "Trends in electronic packaging and assembly for potable consumer products," in Proceedings of the 3rd Electronics Packaging Technology Conference, pp. 24-32, December 2000.

[2] M.-J. Yim and K.-W. Paik, "Design and understanding of anisotropic conductive films (ACF's) for LCD packaging," IEEE Transactions on Components Packaging and Manufacturing Technology A, vol. 21, no. 2, pp. 226-234, 1998.

[3] H. Kristiansen and J. Liu, "Overview of conductive adhesive interconnection technologies for LCD's," IEEE Transactions on Components Packaging and Manufacturing Technology A, vol. 21, no. 2, pp. 208-214, 1998.

[4] J. Liu, A. Tolvgârd, J. Malmodin, and Z. Lai, "A reliable and environmentally friendly packaging technology-flip-chip joining using anisotropically conductive adhesive," IEEE Transactions on Components and Packaging Technologies, vol. 22, no. 2, pp. 186-190, 1999.

[5] I. Watanabe, Y. Gotoh, and K. Kobayashi, "Packaging technologies using anisotropic conductive adhesive films in FPDs," in Proceedings of the 21st International Display Research Conference in Conjunction with the 8th International Display Workshops (Asia Display/IDW '01), pp. 553-556, Nagoya, Japan, October 2001.

[6] P. Savolainen, I. Saarinen, and O. Rusanen, "High-density interconnections in mobile phones using ACF," in Proceedings of the 4th IEEE International Conference on Polymers and Adhesives in Microelectronics and Photonics, pp. 99-104, Portland, Ore, USA, September 2004.

[7] K. Lee, H. J. Kim, I. Kim, and K. W. Paik, "Ultrasonic anisotropic conductive films (ACFs) bonding of flexible substrates on organic rigid boards at room temperature," in Proceedings of the 57th Electronic Components and Technology Conference (ECTC '07), pp. 480-486, Reno, Nev, USA, June 2007.

[8] K. Lee, I. J. Saarinen, L. Pykari, and K. W. Paik, "High power and high reliability flex-on-board assembly using solder anisotropic conductive films combined with ultrasonic bonding technique," IEEE Transactions on Components, Packaging and Manufacturing Technology, vol. 1, no. 12, pp. 1901-1907, 2011.

[9] M. N. Tolunay, P. R. Dawson, and K. K. Wang, "Heating and bonding mechanisms in ultrasonic welding of thermoplastics," Polymer Engineering and Science, vol. 23, no. 13, pp. 726-733, 1983.

[10] C. J. Nonhof and G. A. Luiten, "Estimates for process conditions during the ultrasonic welding of thermoplastics," Polymer Engineering and Science, vol. 36, no. 9, pp. 1177-1183, 1996.

[11] Y. W. Chiu, Y. C. Chan, and S. M. Lui, "Study of shortcircuiting between adjacent joints under electric field effects in fine pitch anisotropic conductive adhesive interconnects," Microelectronics Reliability, vol. 42, no. 12, pp. 1945-1951, 2002.
[12] K. L. Suk, "Effects of nanofiber materials of nanofiber anisotropic conductive adhesives (nanofiber ACAs) for ultrafine pitch electronic assemblies," in Proceedings of the 63rd IEEE Electronic Components and Technology Conference, pp. 118-123, Las Vegas, Nev, USA, May 2013.

[13] S. H. Lee, K. L. Suk, and K. W. Paik, "Study on fine pitch flexon-flex assembly using nanofiber/solder anisotropic conductive film and ultrasonic bonding method," IEEE Transactions on Advanced Packaging, vol. 2, no. 12, pp. 2108-2114, 2012.

[14] K. L. Suk, C. K. Chung, and K. W. Paik, "Effects of nanofiber on the electrical properties of anisotropic conductive adhesives (ACAs)," Journal of Nanoscience and Nanotechnology, vol. 13, no. 1, pp. 351-355, 2013.

[15] K. L. Suk, J. H. Han, J. Y. Lee, and K.-W. Paik, "Advancing electronic packaging using microsolder balls: making $25-\mathrm{nm}$ pitch interconnection possible," IEEE Nanotechnology Magazine, vol. 7, no. 1, pp. 24-30, 2013.

[16] Z.-M. Huang, Y.-Z. Zhang, M. Kotaki, and S. Ramakrishna, "A review on polymer nanofibers by electrospinning and their applications in nanocomposites," Composites Science and Technology, vol. 63, no. 15, pp. 2223-2253, 2003.

[17] D. Li and Y. Xia, "Electrospinning of nanofibers: reinventing the wheel?" Advanced Materials, vol. 16, no. 14, pp. 1151-1170, 2004.

[18] K. Gao, X. Hu, C. Dai, and T. Yi, "Crystal structures of electrospun PVDF membranes and its separator application for rechargeable lithium metal cells," Materials Science and Engineering B, vol. 131, no. 1-3, pp. 100-105, 2006.

[19] H. Na, Y. Zhao, X. Liu, C. Zhao, and X. Yuan, "Structure and properties of electrospun poly(vinylidene fluoride)/polycarbonate membranes after hot-press," Journal of Applied Polymer Science, vol. 122, no. 2, pp. 774-781, 2011. 

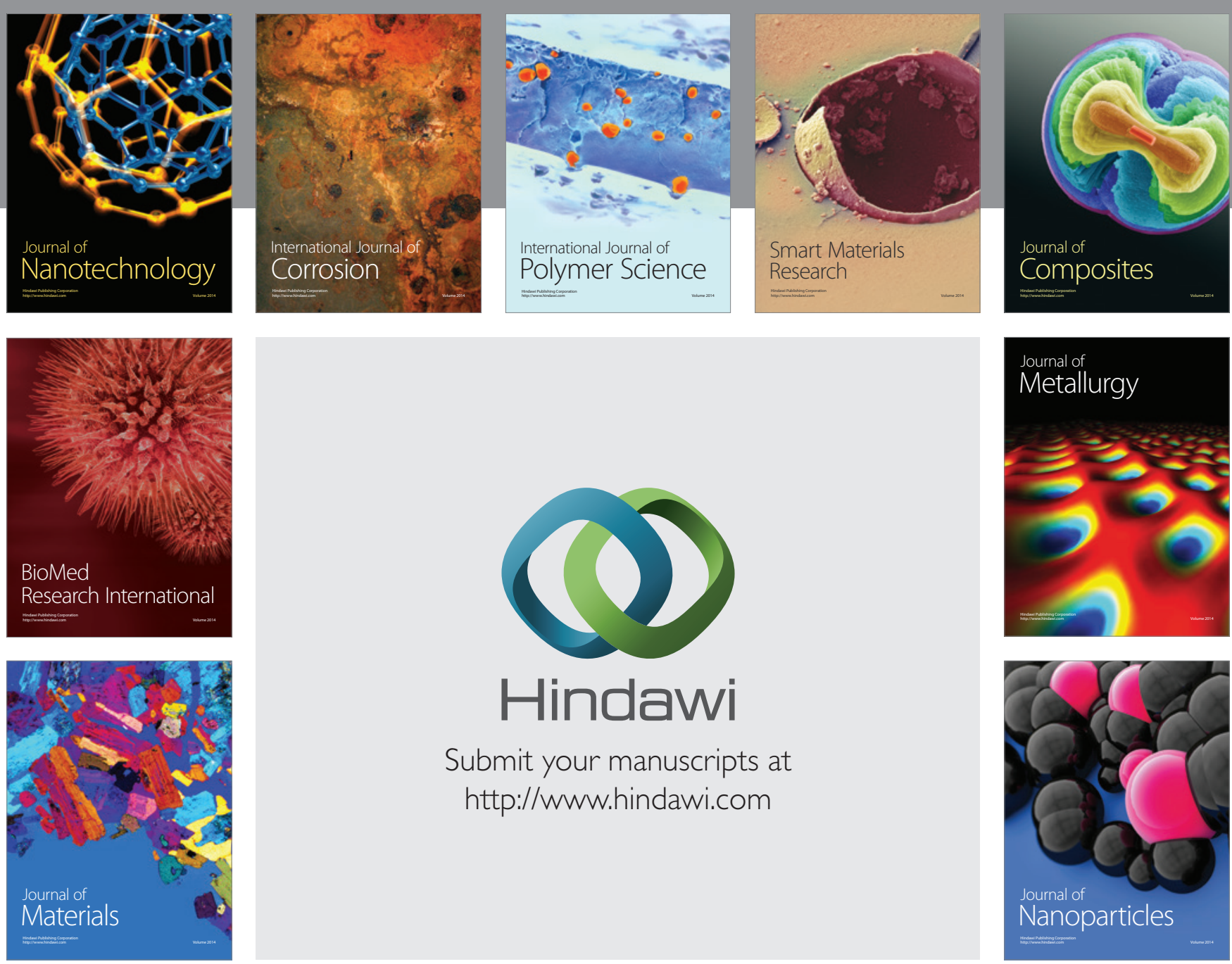

Submit your manuscripts at http://www.hindawi.com
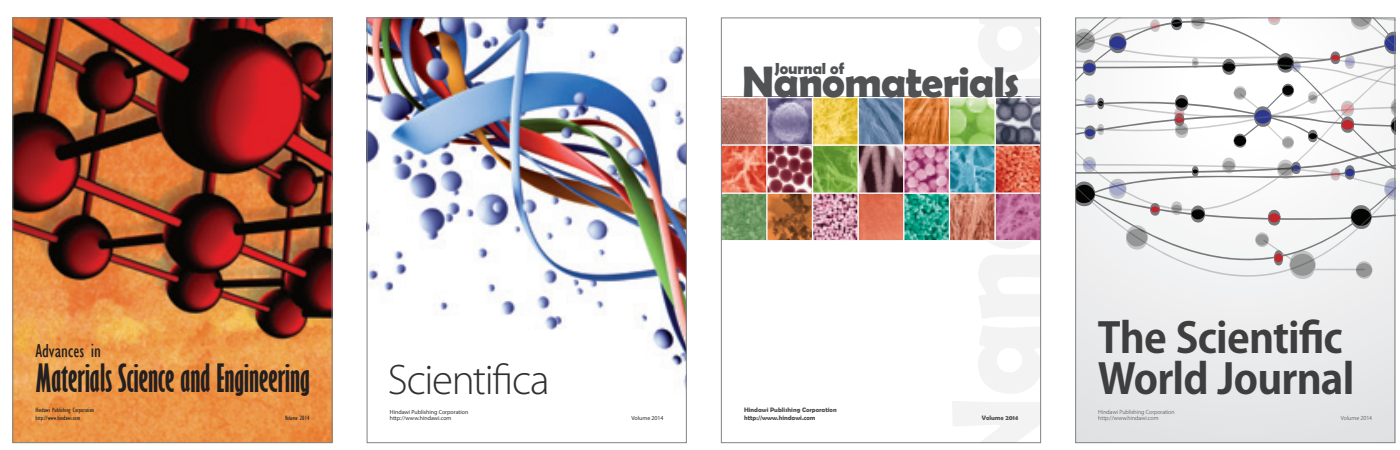

\section{The Scientific World Journal}
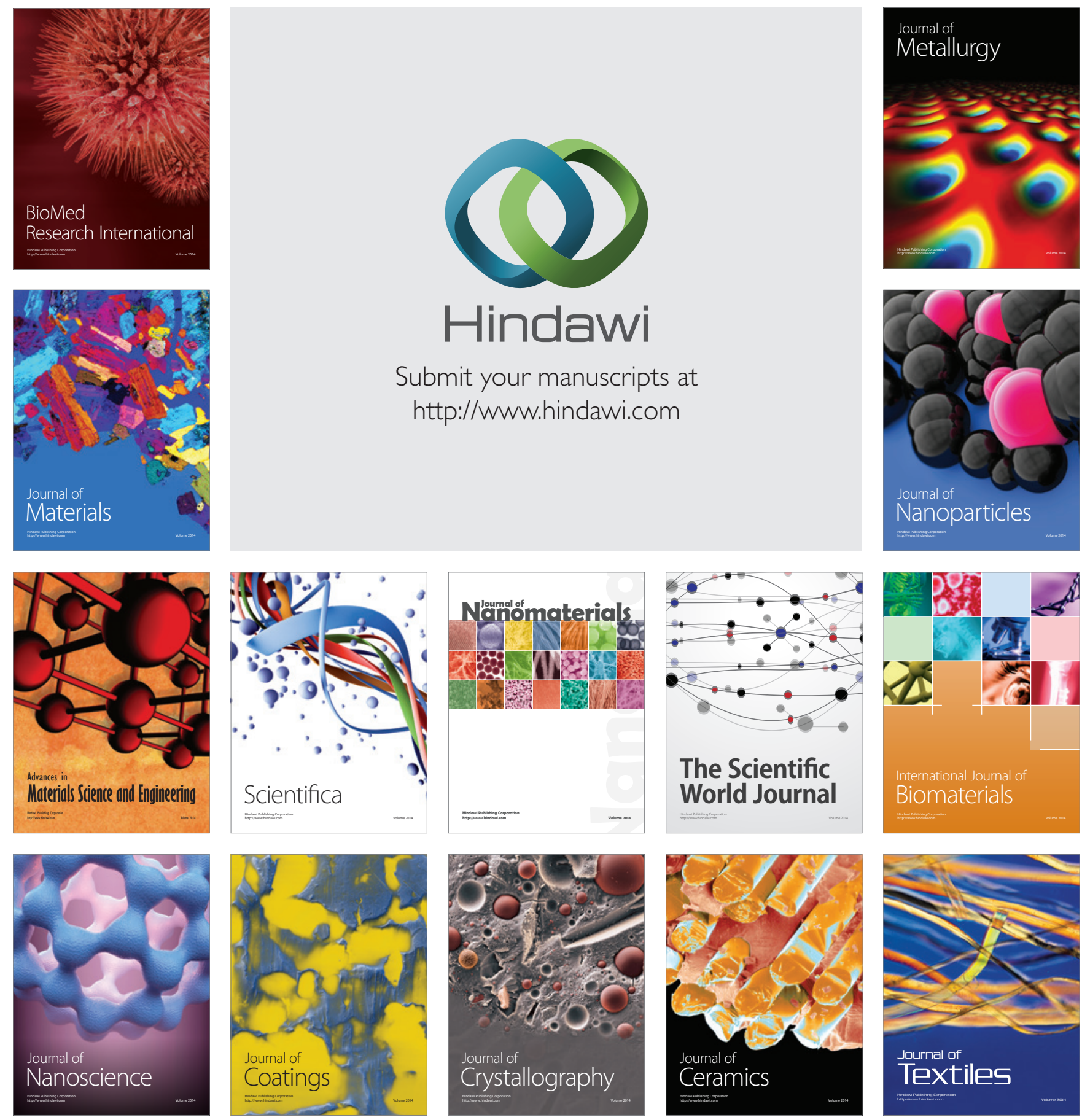